Supporting Information

\title{
Living DNA Nanogel Appendant enables in situ Modulation and Quantification of Regulation Effects on Membrane Proteins
}

Juan Song ${ }^{1 . t}$, Yinan Shen ${ }^{3 . t}$, Han-Zhang Mou, ${ }^{1.7}$ Wen $^{2} i^{2}$, Julie Brouchon ${ }^{3}$, Bi-Yi Xu, ${ }^{2 *}$ Xing-Hua Xia ${ }^{1}$, Jing-Juan Xu ${ }^{1}$ and Hong-Yuan Chen ${ }^{1 *}$

${ }^{1}$ State Key Laboratory of Analytical Chemistry for Life Science, School of Chemistry and Chemical Engineering, Nanjing University, Nanjing, 210023, China. E-mail: hychen@nju.edu.cn

${ }^{2}$ Laboratory of Polymer Chemistry, Department of Polymer Materials, College of Materials Science and Engineering, Shanghai University, Shanghai, 200444, China.

E-mail: xubiyi@shu.edu.cn

${ }^{3}$ Department of Physics, School of Engineering and Applied Sciences, Harvard University Cambridge, MA 02138, USA.

*Corresponding authors.

E-mail: xubiyi@shu.edu.cn, Tel/Fax: +86-21-66138044

E-mail: hychen@nju.edu.cn, Tel/Fax: +86-25-89684862

\section{Table of Contents :}

Evaluation of LCRCA Efficiency by Gel Electrophoresis.

Evaluation of Cytotoxicity of LCRCA in the Solution

Validation of LCRCA of Integrin and EpCAM Targeted Appendants

LCRCA of Mismatch on PC3 Cells

Analysis of the Red/Green Ratio of Pixels on the Images S-4

Analysis of Distributions of the Actins S-4 


\section{Evaluation of LCRCA Efficiency by Gel Electrophoresis}

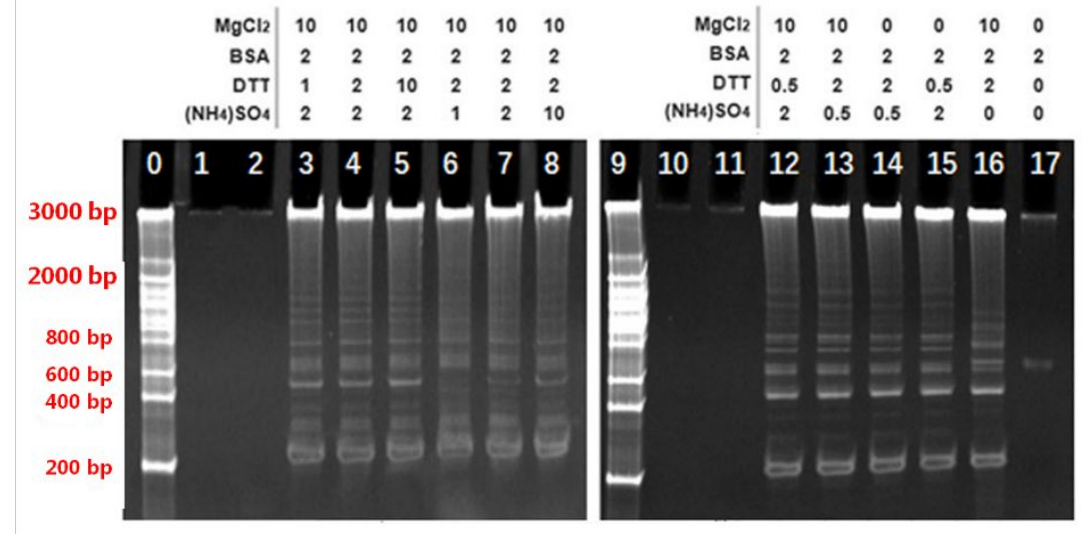

Figure S1. Electrophoresis evaluation of DTT and ammonium sulphate on the efficiency of the LCRCA process. The unit for the concentration of the contents in the inserted tables is $\mathrm{mM}$.

In the commercial kit, the buffer for the RCA reaction contains four ingredients: they are the magnesium chloride, the BSA, the DTT, and the ammonium sulphate. The last two components are poisonous to the cells, with a recommended concentration of $10 \mathrm{mM}$; thus, it is necessary to reduce their content for LCRCA reaction. To evaluate the relationship between their concentration and the efficiency of the RCA reaction, the electrophoresis for the products were taken as shown in Figure S1. The cell culture media was spiked with the ingredients as the buffer for the LCRCA reactions.

\section{Evaluation of Cytotoxicity of LCRCA in the Solution}

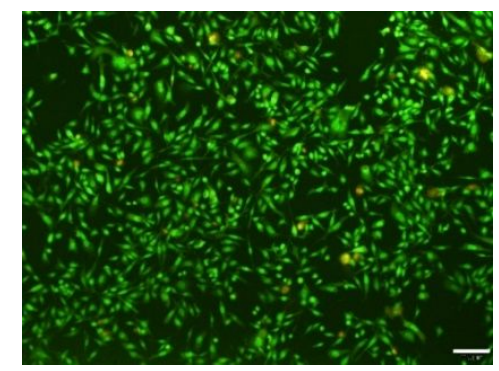

Figure S2. Cytotoxicity evaluation of LCRCA in the solution. (PC3 cells, AO-EB assay, the scale bar equals $100 \mu \mathrm{m}$ )

To test the influence of the LCRCA reaction for the cell, we carried out AO/EB assay to evaluate the viabilities of cells subject to LCRCA in the solution. The LCRCA only occurs to DNAs not attached to the cell membrane. We exposed the cells to the optimized LCRCA solution and carried on the LCRCA process for $1 \mathrm{~h}$ before the AO/EB assay. In AO/EB assay, dead cells emit red fluorescence, and the living cells emit green fluorescence. As shown in Figure S2, more than $95 \%$ of cells were green and maintained polarized shape, implying the cells can maintain a good state with the LCRCA reaction in the solution. 


\section{Validation of LCRCA of Integrin and EpCAM Targeted Appendants}

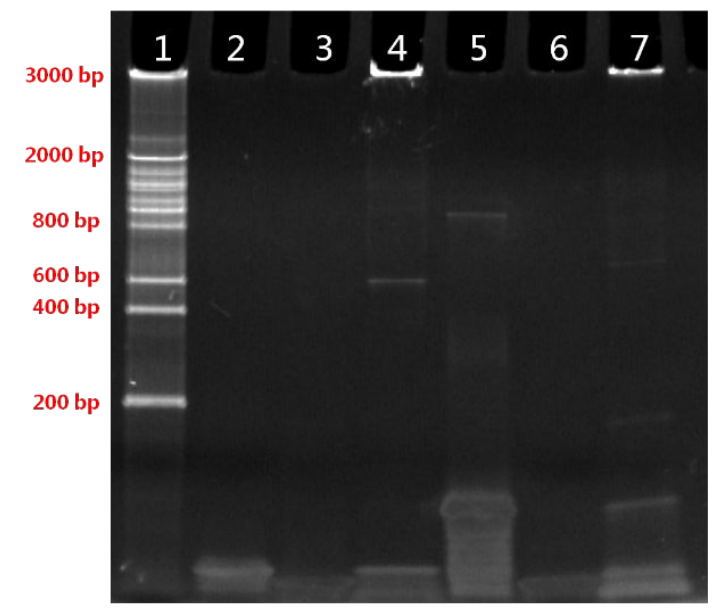

Figure S3. Validation of Integrin-aptamer and EpCAM aptamer-based LCRCA in vitro. (1. DNA marker, 2. Integrin-aptamer, 3. Circular DNA precursor for integrin, 4. LCRCA products-Integrin, 5. EpCAM-aptamer, 6. Circular DNA precursor for EpCAM, and 7. LCRCA products- EpCAM).

To ensure that the designed aptamer-initiator-probe can successfully trigger the RCA reaction, in vitro electrophorese for verification was performed. The electrophoresis results are shown in Figure S3. The results confirmed that the LCRCA for both Integrin and EpCAM are workable.

\section{LCRCA of Mismatch on PC3 Cells}
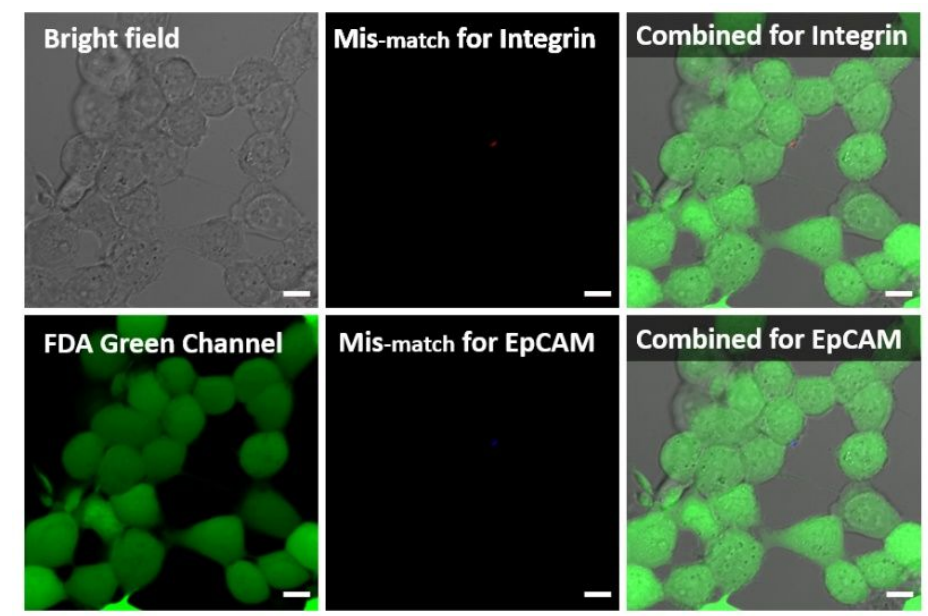

Figure S4. CLSM image of PC3 cells subject to mismatch tags treatment after $30 \mathrm{~min}$ of LCRCA on both Integrin and EpCAM. (The scale bars are all $10 \mu \mathrm{m}$ ).

Figure S4 indicate the mismatch can hardly attach to the ssDNA products on the cell membrane proteins, thus have a negligible contribution to the fluorescence signals. 


\section{Analysis of the Red/Green Ratio of Pixels on the Images}

The images were loaded to the Matlab as an RGB matrix, and the cut-off values that represent the red $(R)$ regions and the green $(G)$ regions were chosen for each image by random sampling. Then the number of pixels above the cut-off values was counted. The ratio was calculated based on the number of the red pixels divided by the green pixels. The influence of the intensity of the color of each pixel was diminished, only the area counts. Thus, the results can better reflect the real distribution of the area covered by the cells, getting rid of the bias from the intensity of the light.

\section{Analysis of Distributions of the Actins}

The Fuji package for Image-J was applied for the generation of the false-colors for visualization of the directions of actin filaments in the cell, and their values were collected with the same package and analyzed using the python algorithm. 
